\title{
Phase transitions and exact ground-state properties of the one-dimensional Hubbard model in a magnetic field
}

\author{
C Yang †, A N Kocharian‡ and Y L Chiang $†$ \\ $\dagger$ Department of Physics, Tamkang University, Tamsui, Taiwan 251, Republic of China \\ \$ Department of Physics and Astronomy, California State University, Northridge, \\ CA 91330-8268, USA \\ $\S$ Department of Physics, Chinese Culture University, Taipei, Taiwan 111, Republic of China
}

Received 3 April 2000

\begin{abstract}
The exact phase diagrams of the one-dimensional Hubbard model, both attractive and repulsive, in the presence of an arbitrary magnetic field $h$ for various electron concentrations $n$ and on-site interaction strengths $U<0$ or $U>0$ are calculated and investigated. The exact ground-state properties, namely, the ground-state energy, the average spin (magnetization), the concentration of the doubly occupied sites, the kinetic energy, the chemical potential, the spin (magnetic) susceptibility and the charge compressibility, are calculated and examined over a wide range of interaction strengths $U$ for various $h$ and $n$. It is found that the spin susceptibility at halffilling is non-analytic and changes discontinuously as $U \rightarrow 0$. The exact theory shows the absence of a charge energy gap in the $U \leqslant 0$ region for all $n$ and provides, for the chemical potential, the rigorous upper and lower bounds for half-filled and empty bands respectively. The analytical results derived for the weak-coupling limit and asymptotic expansions for strong coupling are in full agreement with the numerical calculations.
\end{abstract}

\section{Introduction}

The superconductivity and magnetism in low-dimensional systems have attracted much interest since the discovery of self-organized stripe arrays, layers and 'ladder' structures in high- $T_{c}$ superconducting (HTSC) copper oxides [1-4]. Many predictions have been made with the use of simplified models of electronic structure to capture the basic mechanisms and essential features of the HTSC materials with highly anisotropic electronic properties. Due to the layered structure, HTSC cuprates interpolate between two- and three-dimensional systems and more recently there was found also explicit evidence [3-6] for local quasi-one-dimensional character of the electronic structure. In the search for a mechanism responsible for the superconductivity in these materials, it is instructive to investigate the variation of the ground-state properties versus magnetic field and interpret the observed magnetic features [7], using a simplified model with a magnetic field.

The Hubbard Hamiltonian with on-site attraction $(U<0)$ or repulsion $(U<0)$ of electrons displays the essential features observed in HTSC materials and forms a reasonable minimal model, where the electron correlation length and character of the pairing can be tuned by varying the interaction strength $|U / t|$ and the sign of the parameter $U$, the magnetic field $h$ and the electron concentration $n$. It also constitutes one of the very few exactly solvable models in one dimension $[8,9]$.

The ground-state properties of the one-dimensional Hubbard model have been analysed for the case where $U<0[9-14]$ and $U>0$ [15-19] for various $n$. Some elementary 
excitations and thermodynamic characteristics, including the specific heat coefficient, have been calculated at finite temperatures [12,20-22]. In the presence of a magnetic field some of the ground-state properties were investigated within the attractive [9-12] and repulsive Hubbard models [9, 12,17-19]. However, only the number of doubly occupied sites has been calculated so far for the entire $U$-space $(-\infty<U<+\infty)$ for the half-filling case [22]. Some additional bibliography on this subject can be found in the review papers [23-25].

The exact theory serves as a benchmark for testing the accuracy of the approximate theories especially in the intermediate-coupling regime $(|U / t| \sim 1)[14,26]$. In order to investigate the electron-electron $(U<0)$ and electron-hole $(U>0)$ coupling versus the pair-breaking effect of the magnetic field and $n$, the dependences of the exact ground-state properties on $U$ over the entire parameter space are very desirable. The purpose of this paper to carry out an exact study over the entire parameter space $-\infty<U<+\infty$ of the competition between the attractive interaction between electrons at $U<0$ or between the electron and hole at $U \geqslant 0$ responsible for the formation of electron-electron and electron-hole bound pairs respectively and the corresponding pair-breaking effect of hole doping and the magnetic field.

Below, we present numerical Bethe-ansatz calculations of the ground-state properties and the phase diagram in the presence of a magnetic field. The critical behaviour near the onset magnetization and magnetic saturation are also analysed. It is found that the compressibility and spin (magnetic) susceptibility exhibit anomalous behaviour and discontinuity as $U / t \rightarrow 0$. The results for the repulsive Hubbard model are equivalent to those obtained for the attractive case and show the exact mapping between the positive- and negative- $U$ Hubbard models $[27,28]$.

The asymptotic expansions in the repulsive case for general densities $n$ were derived earlier $[29,30]$. Here, for comparison with the numerical results we present also new analytical results for $U>0$ and $U<0$ for some limiting cases and $h \neq 0$.

The paper is organized as follows. After an introduction (section 2) we give a short review of the Bethe-ansatz formalism in the presence of a magnetic field (section 3) and the solution of the Lieb-Wu equations for the limiting cases of $U \rightarrow 0$ and the strong-interaction limits, $|U| \rightarrow \infty$ (section 4). In section 5 the magnetization curves are discussed in detail. Section 6 presents the numerical results for some important ground-state properties, including the compressibility and the spin susceptibility. Section 7 constitutes a summary. In the appendices we deal with the expansion scheme for the limit $|U| \rightarrow \infty$ and derive the general expression for the spin susceptibility.

\section{Hubbard model}

The model under consideration is the one-dimensional Hubbard model in the presence of a magnetic field, defined by the Hamiltonian

$H_{h} \equiv H+H_{\mathrm{Z}}=-t \sum_{\langle i, j\rangle, \sigma} c_{i \sigma}^{+} c_{j \sigma}+U \sum_{i} c_{i \uparrow}^{+} c_{i \downarrow}^{+} c_{i \downarrow} c_{i \uparrow}-\frac{h}{2} \sum_{i}\left(c_{i \uparrow}^{+} c_{i \uparrow}-c_{i \downarrow}^{+} c_{i \downarrow}\right)$.

The notation is the same as in [14]. The parameter $U$ is considered to be negative $(U<0$, attractive model) or positive ( $U>0$, repulsive model). The last term, $H_{\mathrm{Z}}$, in equation (1) describes the Zeeman energy in the external magnetic field $H_{\mathrm{mag}}=h / g \mu_{\mathrm{B}}$, where $g \approx 2$ is the electron $g$-factor and $\mu_{\mathrm{B}}$ is the Bohr magneton.

The Hamiltonian (1) commutes with the operators of the total numbers of electrons with up or down spins:

$$
N_{\sigma} \equiv \sum_{i} c_{i \sigma}^{+} c_{i \sigma} \quad \sigma= \pm 1(\uparrow \text { or } \downarrow) .
$$


So the conserving quantities are: the number of electrons $n$ per lattice site (electron concentration or band filling) and the total spin $s$ (magnetization) per lattice site defined as

$$
\begin{aligned}
& n \equiv \frac{1}{N_{\text {latt }}} \sum_{i, \sigma}\left\langle c_{i \sigma}^{+} c_{i \sigma}\right\rangle \\
& s \equiv \frac{1}{2 N_{\text {latt }}} \sum_{i, \sigma} \sigma\left\langle c_{i \sigma}^{+} c_{i \sigma}\right\rangle
\end{aligned}
$$

where $N_{\text {latt }}$ is the total number of lattice sites, $0 \leqslant n \leqslant 1,0 \leqslant s \leqslant n / 2$. The electron concentration $n$ is connected with the doping $\delta_{\text {dop }}=1-n$. The limiting cases $n=0$ and $n=1$ correspond to empty and half-filled bands respectively.

It is convenient to determine the ground-state average spin $s$ in an external magnetic field $h$ by minimizing the average energy $E_{h} \equiv\left\langle H_{h}\right\rangle / N_{\text {latt }}$, i.e. from the equation

$$
h=\frac{\partial E}{\partial s}
$$

where $E \equiv\left\langle H_{h}-H_{\mathrm{Z}}\right\rangle / N_{\text {latt }}$.

\section{Lieb-Wu equations for $U>0$ and $U<0$}

The Bethe-ansatz technique $[8,11,13]$ gives an exact solution of the Hubbard model with the Hamiltonian $H$ in one dimension. In the limit $N_{\text {latt }} \gg 1$ the corresponding expressions for the ground-state characteristics in the attractive case $(U<0)$ were given in [14]. In the general (attractive or repulsive) case the energy of the system per lattice site is

$$
E=U\left(\frac{n}{2}-s\right) \Theta(-U)-2 t \int_{-Q}^{Q} \mathrm{~d} k \rho(k) \cos k
$$

$\Theta(x)=1$ if $x>0$ and $\Theta(x)=0$ if $x<0$, and $\rho(k), \sigma(\lambda), f_{1}(k, \lambda), f_{2}\left(\lambda, \lambda^{\prime}\right)$ are determined from equations (19)-(22) of [14], where $U$ must be replaced by $|U|$. The normalization conditions are

$$
\int_{-Q}^{Q} \rho(k) \mathrm{d} k=(1-2 s) \Theta(-U)+n \Theta(U) \quad \int_{-B}^{B} \sigma(\lambda) \mathrm{d} \lambda=\frac{n}{2}-s .
$$

The expectation value of the Hamiltonian $H_{h}$ per lattice site is

$$
E_{h}=E-h s .
$$

The numerical solutions for $U<0$ and general $n$ and $s=0$ have been reported earlier in references $[13,14,26]$. The solution of the corresponding Bethe-ansatz equations determines the energies $E$ and $E_{h}$ for general values of $U / t, h$ (or $s$ ) and $n$.

\section{Limiting cases}

\subsection{Weak-coupling limit}

Here we consider analytical results in the exact theory for some limiting cases. For comparison we present here results for $U>0$ along with the $U<0$ case.

In the limit $|U / t| \rightarrow 0$ the functions $f_{1}(k, \lambda)$ and $f_{2}\left(\lambda, \lambda^{\prime}\right)$ have sharp peaks (see equations (21) and (22) in [14]) at $\lambda=\sin k$ and $\lambda=\lambda^{\prime}$ respectively. With an accuracy 
of the order of $|U| / t$ or higher, we have an explicit solution of the coupled integral equations for $\rho(k)$ and $\sigma(\lambda)$ for general electron concentration and arbitrary magnetic field:

$$
\begin{aligned}
& \rho(k)= \begin{cases}1 / \pi & \text { for }|\sin k|<B \\
1 / 2 \pi & \text { for }|\sin k|>B\end{cases} \\
& \sigma(\lambda)=\frac{1}{2 \pi \sqrt{1-\lambda^{2}}} \quad \text { for }-B<\lambda<B
\end{aligned}
$$

and

$$
Q=\left(1-\frac{n}{2}-s\right) \pi \quad B=\sin \left[\left(\frac{n}{2}-s\right) \pi\right]
$$

Using (9) and (11) we have

$$
E=-\frac{4 t}{\pi} \sin \left(\frac{n \pi}{2}\right) \cos (s \pi)
$$

The magnetization in the limiting case $U \rightarrow 0$ is found from equations (5) and (12):

$$
s= \begin{cases}\frac{1}{\pi} \arcsin \frac{h}{4 t \sin (n \pi / 2)} & \text { for } 0 \leqslant h \leqslant h_{\mathrm{c} 2} \\ n / 2 & \text { for } h_{\mathrm{c} 2}<h\end{cases}
$$

and the spin (magnetic) susceptibility is

$$
\chi \equiv \frac{t \partial s}{\partial h}= \begin{cases}\frac{1}{4 \pi \sin (n \pi / 2) \cos (s \pi)} & \text { for } 0 \leqslant h \leqslant h_{\mathrm{c} 2} \\ 0 & \text { for } h_{\mathrm{c} 2}<h\end{cases}
$$

where

$$
h_{\mathrm{c} 2}=4 t \sin ^{2}(n \pi / 2)
$$

is a critical value of $h$ for spin saturation (see (13) and below, section 5.1).

It is also easy to get an explicit formula for the concentration of the doubly occupied sites (see section 5.2):

$$
D=\frac{\partial E}{\partial U}=\frac{n^{2}}{4}-s^{2}
$$

and for the kinetic energy (see section 5.3):

$$
E_{\mathrm{kin}}=\frac{t \partial E}{\partial t}=E
$$

and the chemical potential (see section 5.4):

$$
\mu=\frac{\partial E}{\partial n}=-2 t \cos \left(\frac{n \pi}{2}\right) \cos (s \pi)
$$

and also the compressibility (see section 5.4):

$$
\kappa_{\mathrm{ch}}=\frac{\partial^{2} E}{\partial n^{2}}=t \pi \sin \left(\frac{n \pi}{2}\right) \cos (s \pi) .
$$




\subsection{Strong-coupling limit}

For given $n$ and $s$ at $|U| \gg 4 t$ we have general expressions for the main values (see appendix A):

$$
\begin{aligned}
& Q= \begin{cases}(1-2 s) \pi & \text { for } U<0 \\
n \pi & \text { for } U>0\end{cases} \\
& B \propto \frac{|U|}{t} .
\end{aligned}
$$

So the main value of the energy is

$$
E= \begin{cases}U\left(\frac{n}{2}-s\right)-\frac{2 t}{\pi} \sin (2 s \pi) & \text { for } U<0 \\ -\frac{2 t}{\pi} \sin (n \pi) & \text { for } U>0 .\end{cases}
$$

From here we get the limiting values

$$
\begin{aligned}
& \mu=\frac{\partial E}{\partial n}= \begin{cases}U / 2 & \text { for } U<0 \\
-2 t \cos n \pi & \text { for } U>0\end{cases} \\
& \kappa_{\mathrm{ch}}=\frac{\partial \mu}{\partial n}= \begin{cases}\mathcal{O}(t / U) & \text { for } U<0 \\
2 \pi t \sin n \pi & \text { for } U>0\end{cases} \\
& D=\frac{\partial E}{\partial U}= \begin{cases}n / 2-s & \text { for } U<0 \\
\mathcal{O}(t / U) & \text { for } U>0\end{cases} \\
& E_{\text {kin }}=t \frac{\partial E}{\partial t}= \begin{cases}-2 t \sin (2 s \pi) / \pi & \text { for } U<0 \\
-2 t \sin n \pi / \pi & \text { for } U>0\end{cases} \\
& h=\frac{\partial E}{\partial s}= \begin{cases}-U-4 t \cos (2 s \pi) & \text { for } U<0 \\
\mathcal{O}(t / U) & \text { for } U>0\end{cases} \\
& \frac{1}{\chi}=\frac{\partial^{2} E}{\partial s^{2}}= \begin{cases}8 \pi t \sin (2 s \pi) & \text { for } U<0 \\
\mathcal{O}(t / U) & \text { for } U>0\end{cases}
\end{aligned}
$$

where $\mathcal{O}(x) \rightarrow 0$ at $|x| \rightarrow 0$.

From the last two formulae (27) and (28) we find that at $U \gg 4 t$ the spin saturation state $(s=n / 2)$ is realized at infinitesimal magnetic field $\left(h_{\mathrm{c} 2} \rightarrow 0\right)$ and the corresponding spin (magnetic) susceptibility $\chi$ diverges at $h \rightarrow 0$.

It is necessary to note that formulae (9)-(28) are obtained at $n \neq 0$. For example, in an empty band $(n=0)$ for $U<0$ the average spin $s \equiv 0$, so every quantity is independent of the magnetic field. In this case we have $B=0, \rho=1 / 2 \pi, Q=\pi$, $E=E_{h}=D=E_{\text {kin }}=\chi=0$, the critical magnetic fields for spin (magnetization) onset and saturation, $h_{\mathrm{c} 1}$ and $h_{\mathrm{c} 2}$ (see section 5.1), are equal to each other for arbitrary coupling and $[12,14,31,32]$

$$
\mu=-\sqrt{\frac{U^{2}}{4}+4 t^{2}}
$$

(see appendix B), while $\mu=-2 t$ for all $U \geqslant 0$ and $h$. 


\section{Spin (magnetization) and the energy gap}

\subsection{Spin (magnetization)}

First we give the description of the dependence of the spin $s$ upon $h$ necessary for understanding the critical behaviour in the ground state. The $s$-versus- $h$ curves (magnetization curves) are found from equation (5) numerically for arbitrary $U / t$ and $n$ (see appendix B).

Our calculations show that the derivative of the energy with respect to the spin $s$ (which determines the magnetic field in equilibrium) is an increasing function of $s$. Let $h_{\mathrm{cl}}(n, U)$ be the solution of equation (5) for the minimal value of the spin $(s=0)$ at given $n$ and $U$. At $h<h_{\mathrm{c} 1}(n, U)$ the spin of the system has the constant value $s=0$ (the zero-spin phase, or phase I). A finite spin (magnetization) appears only if the magnetic field exceeds the critical field $h_{\mathrm{c} 1}(n, U)$ (figure 1, upward-pointing solid triangles), associated with the spin energy gap, and there is no paramagnetic response in the system to the smaller field $h \leqslant h_{\mathrm{c} 1}(n, U)$. In the repulsive case the spin energy gap does not exist, so $h_{\mathrm{c} 1}(n, U) \equiv 0$ independently of however strong $U>0$ is and an infinitesimal $h$ results in finite $\chi^{-1}$ for all $n$ (see section 6.5).

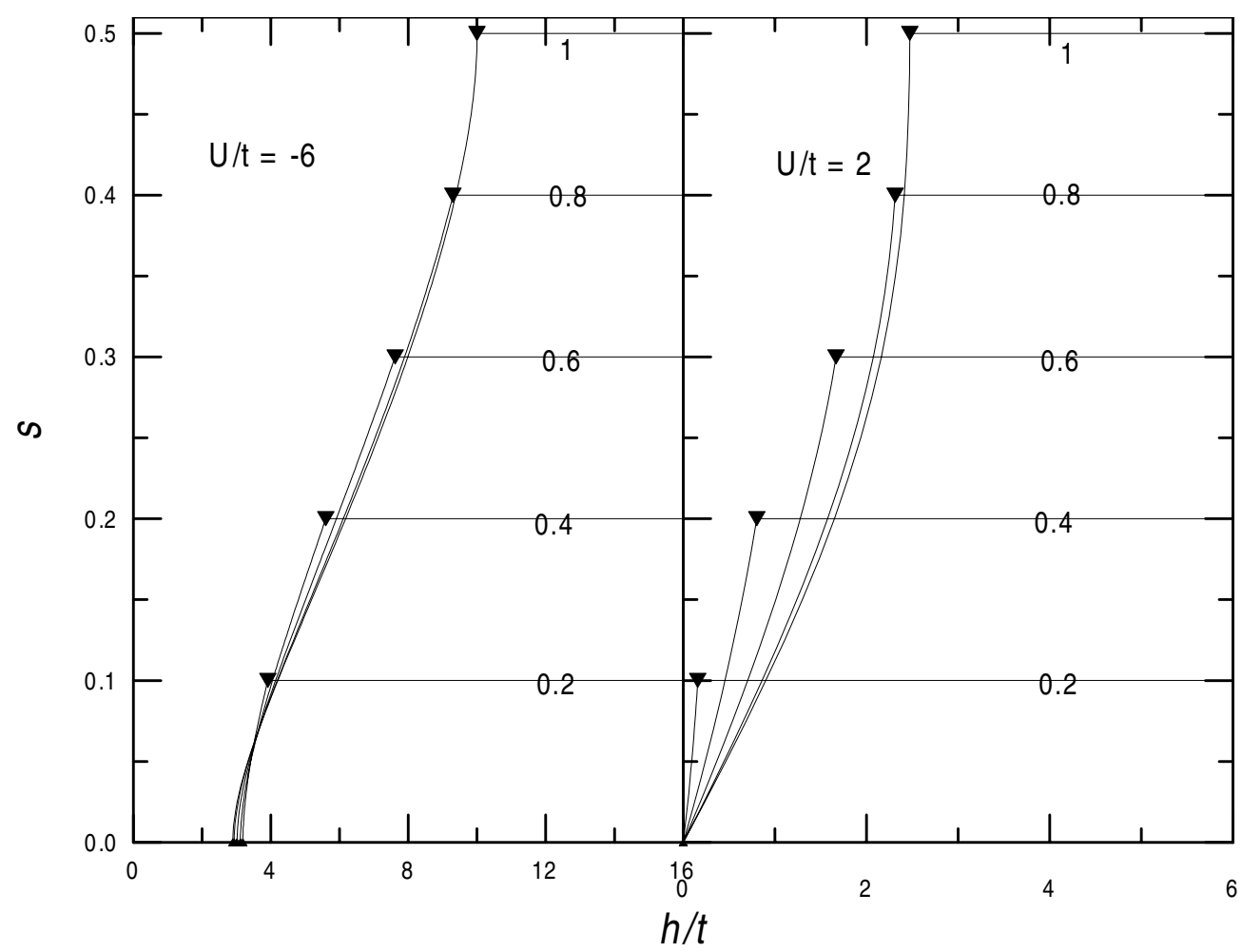

Figure 1. The average spin (magnetization) $s$ versus magnetic field $h$ in the attractive $(U=-6 t)$ and repulsive $(U=2 t)$ Hubbard models for various electron concentrations $n$ (figures labelling the curves). The upward-pointing and downward-pointing triangles mark the critical magnetic field values $h_{\mathrm{c} 1} / t$ and $h_{\mathrm{c} 2} / t$ respectively.

Let $h_{\mathrm{c} 2}(n, U)$ (figure 1, downward-pointing solid triangles) be the solution of equation (5) for the maximal value of the spin $s=n / 2$ at given $n$ and $U$. For $h_{\mathrm{c} 1}(n, U)<h<h_{\mathrm{c} 2}(n, U)$ the spin of the system is finite, but not saturated, $0<s<n / 2$ (intermediate phase or phase II). Finally, for $h>h_{\mathrm{c} 2}(n, U)$ the saturated spin has the constant value $s=n / 2$ (spin saturation 
phase or phase III).

Expanding the energy $E$ into a power series in $s$ in the vicinity of $h_{\mathrm{c} 1}$ (near the onset of magnetization) and taking the partial derivative of $E$ with respect to $s$ at $s=0$, we have a simple relation between the spin (magnetization) and magnetic field:

$$
h-h_{\mathrm{c} 1}=a_{1} s+a_{2} s^{2}+\cdots
$$

where

$$
a_{1} \equiv \partial^{2} E /\left.\partial s^{2}\right|_{s=0} \quad a_{2} \equiv \partial^{3} E /\left.2 \partial s^{3}\right|_{s=0} .
$$

When $a_{1} \neq 0$ we have a linear dependence:

$$
s=\left(h-h_{\mathrm{c} 1}\right) / a_{1}
$$

and the spin (magnetic) susceptibility is finite

$$
\chi=t / a_{1} .
$$

When $a_{1}=0$ and $a_{2}>0$ we have a square-root dependence:

$$
s=\left(\frac{h-h_{\mathrm{c} 1}}{a_{2}}\right)^{1 / 2}
$$

and the spin (magnetic) susceptibility has a square-root singularity:

$$
\chi=\frac{t}{2\left[a_{2}\left(h-h_{\mathrm{c} 1}\right)\right]^{1 / 2}} .
$$

Analogously, in the vicinity of $h_{\mathrm{c} 2}(n, U)$ we have

$$
s=n / 2-\left(h_{\mathrm{c} 2}-h\right) / b_{1} \quad \chi=-t / b_{1}
$$

if $b_{1} \neq 0$ and

$$
s=\frac{n}{2}-\left(\frac{h_{\mathrm{c} 2}-h}{b_{2}}\right)^{1 / 2} \quad \chi=-\frac{t}{2\left[b_{2}\left(h_{\mathrm{c} 2}-h\right)\right]^{1 / 2}}
$$

if $b_{1}=0$ and $b_{2}>0$, where

$$
b_{1}=-\partial^{2} E /\left.\partial s^{2}\right|_{s=n / 2} \quad b_{2}=-\partial^{3} E /\left.2 \partial s^{3}\right|_{s=n / 2} .
$$

The calculations of the second derivatives of $E$ with respect to $s$ (the inverse spin susceptibility at $h_{\mathrm{c} 1}$ and $h_{\mathrm{c} 2}$, or $a_{1}$ and $b_{1}$ respectively) show that away from half-filling the spin (magnetic) susceptibility at $h_{\mathrm{c} 1}$ and $h_{\mathrm{c} 2}$ is always finite (see also section 6.5). Square-root dependences at $U<0$, equations (33) and (36), for arbitrary $|U| / t$ occur only at $n=1$ and $n=0$. For all $U>0$ the square-root behaviour (36) occurs only at $n=1$ near the saturation $h_{\mathrm{c} 2}(s=1 / 2)$. At $n \approx 1$ the coefficient $a_{1}$ for $U<0$ is very small $\left(a_{1} \leqslant 10^{-3}\right)$, so the linear part (31) is very short. For strong attraction, $a_{1}$ approaches zero for all $n$-values, which gives a large slope at $h_{\mathrm{c} 1}$. This circumstance can explain the discrepancy between the interpretations of the results for magnetization in references $[11,12]$. Thus our result for all $0<n<1$ suggests linear scaling according to equations (31) and (35). It is interesting to note that at the limit of large $-U / t$ and for strong magnetic field, near $h \rightarrow h_{\mathrm{c} 1}$, the parameters $a_{1} \neq 0$ and $b_{1} \neq 0$ and the normalized magnetization $s / n$ give for all $n \neq 1$ a scaling different from that of an isotropic Heisenberg model with magnetic field [33,34].

The square-root behaviour (36) near the spin (magnetic) saturation at $h=h_{\mathrm{c} 2}$ exactly at half-filling for all $U$-values, including $U=0$, reflects the Van Hove singularity near the band edge in one dimension. In addition, at $n=1$ we have similar square-root behaviour, equations (33) and (34), for all $U<0$ near the onset of magnetization at $h_{\mathrm{c} 1}$. In the repulsive region $(U>0), h_{\mathrm{c} 1} \equiv 0$ and an infinitesimal $h$ results in finite $s \neq 0$. 
For fixed $h / t$ and $n$, the spin (magnetization) is zero (phase I) when the attraction is sufficiently strong, i.e. $|U|(U<0)$ is greater than some critical value $\left|U_{\mathrm{c} 1}(n, h)\right|$ $\left(U_{\mathrm{c} 1}(n, h)<0\right)$ (figure 2, upward-pointing solid triangles). If the attraction is weak or the repulsion is strong, i.e. $U$ is greater algebraically than some another critical value $U_{\mathrm{c} 2}(n, h)$ (figure 2, downward-pointing solid triangles) the spin is saturated ( $s=n / 2$, phase III). For intermediate coupling $U_{\mathrm{c} 1}(n, h)<U<U_{\mathrm{c} 2}(n, h)$, the spin is non-zero and unsaturated $(0<s<n / 2$, phase II) and increases algebraically with $U$.

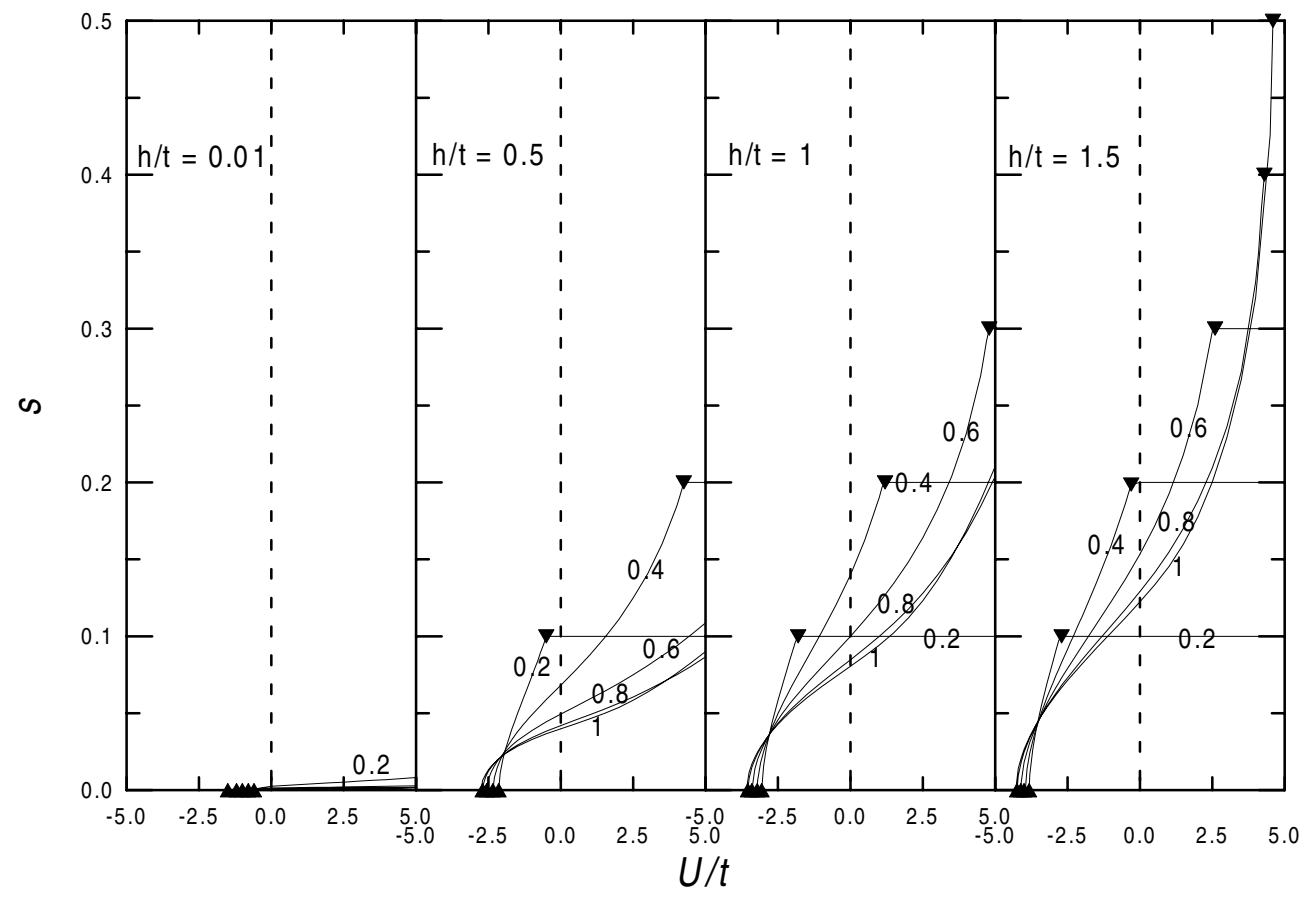

Figure 2. The average spin (magnetization) $s$ versus interaction strength $U / t$ for various magnetic fields $h / t$ and electron concentrations $n$ (figures labelling the curves). The upward-pointing and downward-pointing triangles mark the critical values $U_{\mathrm{c} 1} / t$ and $U_{\mathrm{c} 2} / t$ respectively.

\subsection{Energy gap and phase diagram}

The magnitude of the critical field $h_{\mathrm{c} 1}(n, U)$ for all $U<0$ is associated with the spin energy gap which depends also on the electron concentration [14].

At half-filling, the spin energy gap $h_{\mathrm{c} 1}(1, U)$ is $[10,11,13]$

$$
h_{\mathrm{c} 1}(1, U)=-U-4 t+8 t \int_{0}^{\infty} \mathrm{d} w \frac{J_{1}(w)}{w(1+\exp (-U w / 2 t))}
$$

where $J_{1}(x)$ is the Bessel function of the first order. For fixed $U$ the energy gap increases with the decreasing of $n$ and for an empty band (the zero-concentration limit) we have the maximal value $[13,14,32]$

$$
h_{\mathrm{c} 1}(0, U)=\sqrt{U^{2}+16 t^{2}}-4 t \text {. }
$$

At arbitrary $n, h_{\mathrm{c} 1}(n, U)$ is a decreasing function of $U$ (figure 3 , the bold curves). For $U \geqslant 0$ there is no spin energy gap, so $h_{\mathrm{c} 1}(n, U) \equiv 0$. 


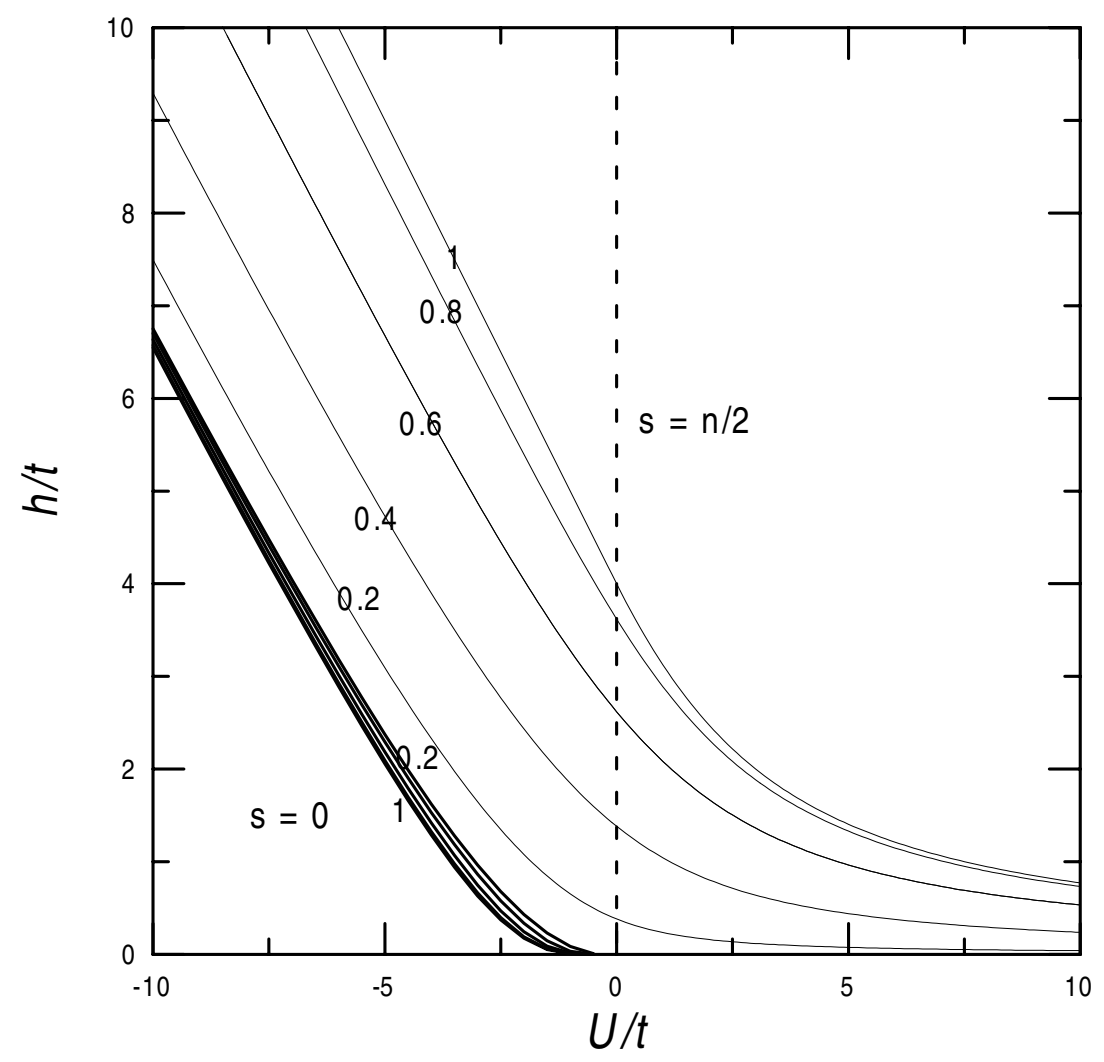

Figure 3. The phase diagram of the one-dimensional Hubbard model. The five thin curves for $n=1,0.8,0.6,0.4,0.2$ (figures labelling the curves) give the critical magnetic field $h_{\mathrm{c} 2} / t$ versus $U / t$ or the critical coupling $U_{\mathrm{c} 2} / t$ versus $h / t$. The five bold curves for $n=0.2,0.4,0.6,0.8,1$ give the critical magnetic field $h_{\mathrm{c} 1} / t$ versus $U / t$ or the critical coupling $U_{\mathrm{c} 1} / t$ versus $h / t$. The region $h \geqslant h_{\mathrm{c} 2}$ (or $U \geqslant U_{\mathrm{c} 2}$ ) corresponds to the saturated spin phase $s=n / 2$. The region $h \leqslant h_{\mathrm{c} 1}$ (or $U \leqslant U_{\mathrm{c} 1}$ ) corresponds to the phase with zero spin, $s=0$. The intermediate area $h_{\mathrm{c} 1} \leqslant h \leqslant h_{\mathrm{c} 2}$ (or $U_{\mathrm{c} 1} \leqslant U \leqslant U_{\mathrm{c} 2}$ ) corresponds to the phase with $0<s<n / 2$.

The number of broken pairs is increased gradually by the applied field and the system becomes fully polarized above the upper critical field $h_{\mathrm{c} 2}(n, U)$. The parameter $h_{\mathrm{c} 2}(n, U)$ at half-filling $(n=1)$ is determined by a simple expression:

$$
h_{\mathrm{c} 2}(1, U)= \begin{cases}4 t-U & \text { for } U<0 \\ \sqrt{U^{2}+16 t^{2}}-U & \text { for } U>0 .\end{cases}
$$

The result for $h_{\mathrm{c} 2}$ at $U \geqslant 0$ was obtained earlier for the closed Hubbard chain and in the thermodynamic limit $[17,35]$. For the general case of a given arbitrary $n$, the upper critical field $h_{\mathrm{c} 2}(n, U)$ is also a decreasing function of $U$ (figure 3 , the thin curves). For given $U$ it decreases with decreasing $n$. At $n=0$ we have a transparent result, $h_{\mathrm{c} 2}(0, U)=h_{\mathrm{c} 1}(0, U)$.

Resolving the equations $h_{\mathrm{c} 1}(n, U)=h$ and $h_{\mathrm{c} 2}(n, U)=h$ with respect to $U$ for given $h$ and $n$, we find the lower and upper critical couplings $U_{\mathrm{c} 1}(n, h)$ and $U_{\mathrm{c} 2}(n, h)$ respectively. It can be seen from figure 3 that $U_{\mathrm{c} 1}(n, h)<0$ for all $n$ and $h$, but $U_{\mathrm{c} 2}(n, h)$ may be positive if $h$ is not very large (e.g., $U_{\mathrm{c} 2}(n, h)>0$ at $h<4 t$ for $\left.n=1\right)$.

Analogously, resolving the equations $h_{\mathrm{c} 1}(n, U)=h$ and $h_{\mathrm{c} 2}(n, U)=h$ with respect to $n$ for given $h$ and $U$, we may obtain correspondingly the first and second critical electron 
concentrations $n_{\mathrm{c} 1}(U, h)$ and $n_{\mathrm{c} 2}(U, h)$. It can be seen from figure 3 that there are only limited areas of values $U$ and $h / t$ where $n_{\mathrm{c} 1}(U, h)$ and $n_{\mathrm{c} 2}(U, h)$ can be found between 0 and 1 .

The curves in figure 3 define the boundaries for the onset and saturation of spin (magnetization) for various $n$-values. The region between the two curves (bold and thin) for the same $n$ corresponds to the intermediate phase of unsaturated spin (magnetization) $0<s<n / 2$.

It is clearly seen from the phase diagram that the increase in magnetic field at $U<0$ is equivalent to a decrease of the interaction strength $|U| / t$ and vice versa. In contrast, for the repulsive case $U>0$, magnetization increases on increasing either $h$ or interaction strength $U$. In strong field $h \geqslant h_{\mathrm{c} 2}$, all electrons are unbound and fully polarized with all spins aligned ( $s=n / 2)$ and, according to equation (12), the energy is the same as for free fermions, $E=-2 t \sin (\pi n) / \pi$. Note that the upper critical field approaches its maximum value at $n=1$ for both cases $U<0$ and $U>0$ [35].

\section{Ground-state properties}

\subsection{Total energy}

The ground-state total energy $E_{h}$ for arbitrary values of interaction strength $U / t$, electron concentration $n$ and spin $s$ can be calculated numerically from the integral equations (see section 3) using the subroutine fred2 (see reference [36], section 18.1).

At given $n$ and $h / t$, the total energy $E_{h}$ monotonically increases (algebraically) with $U / t$ at $U<U_{\mathrm{c} 2}$ (values $U_{\mathrm{c} 2} / t$ are indicated by downward-pointing solid triangles) and this curve has different slopes (figure 4). In phase I (at $U<U_{\mathrm{c} 1}$; values $U_{\mathrm{c} 1} / t$ are indicated by

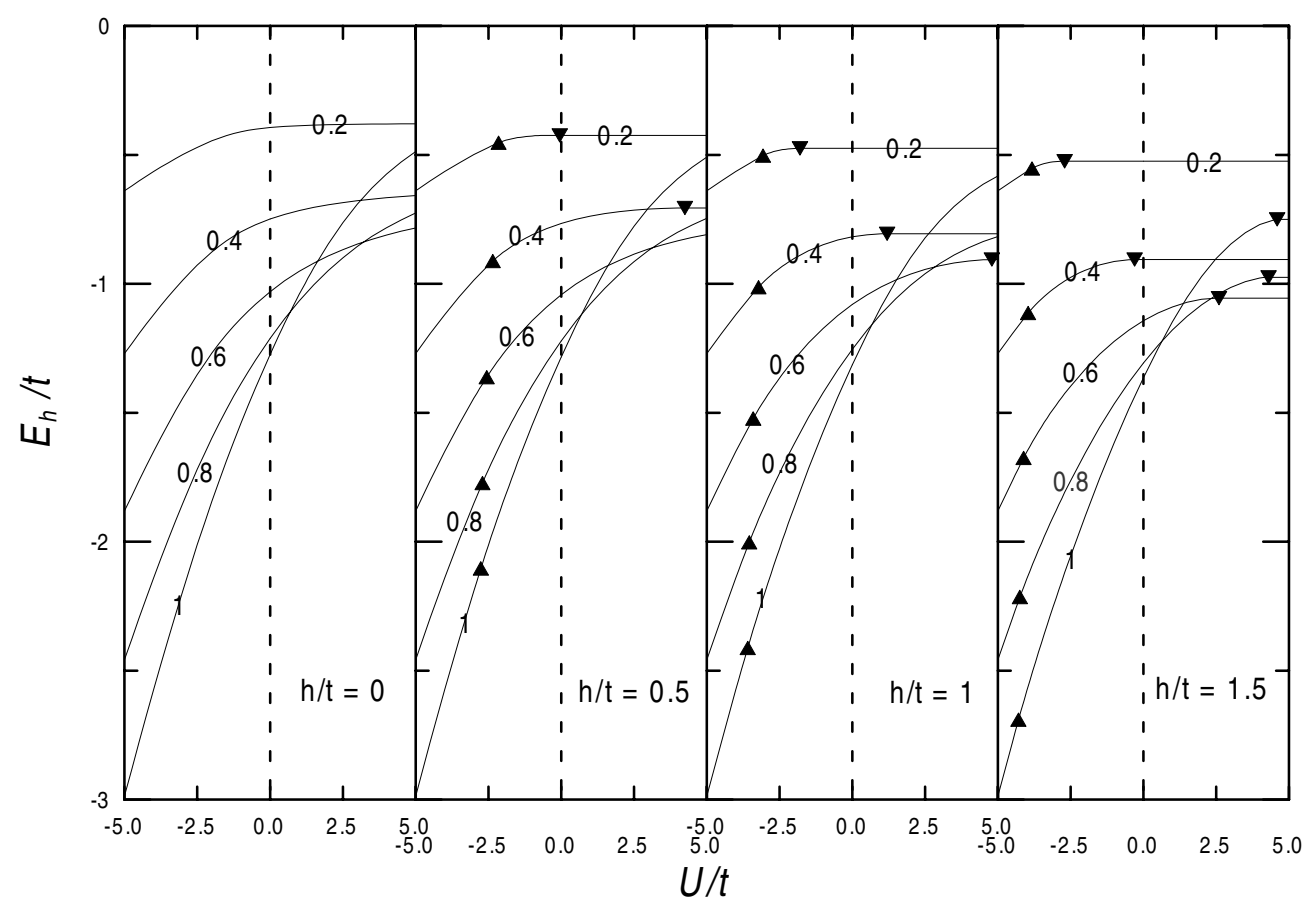

Figure 4. The total ground-state energy $E_{h} / t$ versus $U / t$. The notation is the same as for figure 2 . 
upward-pointing solid triangles) the dependence of $E_{h}$ on $U / t$ is asymptotically linear. In phase II the slope gradually becomes gentler. Finally, at $U>U_{\mathrm{c} 2}$ (values $U_{\mathrm{c} 2} / t$ are indicated by downward-pointing solid triangles) in phase III of saturated spin we have $E_{h}=-h n / 2-2 t \sin (n \pi) / \pi$, independently of $U / t$.

With increasing of $h / t$ at given $U$ and $n$, the total energy $E_{h}$ decreases algebraically. At fixed $h, E_{h}$ versus $n$ is a monotonic function for $U<0$ and it becomes non-monotonic for $U>0$.

\subsection{Concentration of doubly occupied sites}

The concentration of the local pairs or doubly occupied sites $D$ is defined as

$$
D \equiv \frac{1}{N_{\text {latt }}} \sum_{i}\left\langle c_{i \uparrow}^{+} c_{i \uparrow} c_{i \downarrow}^{+} c_{i \downarrow}\right\rangle .
$$

Obviously (see (1)), this quantity measures the derivative of the energy with respect to $U$ :

$$
D=\frac{\partial\langle H\rangle}{N_{\text {latt }} \partial U}=\frac{\partial E}{\partial U}
$$

Using the Lieb-Wu equations (see section 3 in [14] and section 3 in this paper) we calculate $D$ according to equation (41) (see appendix C).

The local attraction of electrons favours the formation of the local pairs with opposite spins, while the local repulsion of electrons suppresses the pair formation. In contrast, the magnetic field acts similarly in both cases and tends to destroy the pairs. So $D$ decreases at given $n$ and $h / t$ by increasing $U / t$ from $-\infty$ (figure 5). At the boundary of the phases I

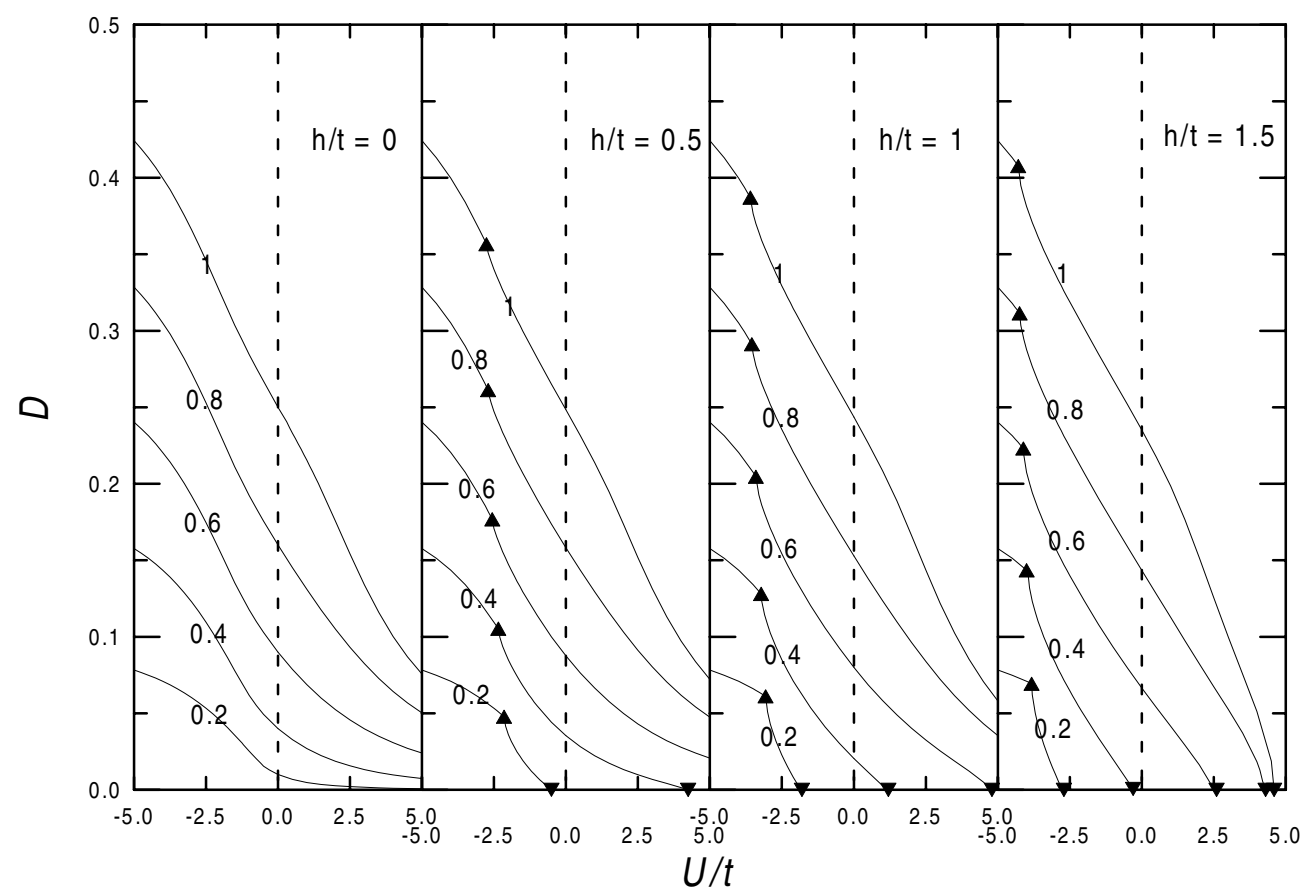

Figure 5. The ground-state concentration of doubly occupied sites $D$ versus $U / t$. The notation is the same as for figure 2 . 
and II, i.e. at the onset of the spin (magnetization) at $U_{\mathrm{cl}}$ (upward-pointing solid triangles), the curve for $D$ versus $U$ has a cusp. Finally, at the boundary of the phases II and III at $U_{\mathrm{c} 2}$ (downward-pointing solid triangles) and above (spin saturation), $D$ becomes zero.

At $n=0$ the concentration of the doubly occupied sites $D$ evidently vanishes for any $U$ and $h$. It vanishes also at $n \leqslant n_{\mathrm{c} 2}$ (phase III with saturated spin) if at given $U$ and $h$ the value $n_{\mathrm{c} 2}$ is found between 0 and 1 . Then $D$ monotonically increases with $n$.

At fixed $U$ and $n$, the magnitude of $D$ is constant at $h \leqslant h_{\mathrm{c} 1}$ (in phase I). However, it decreases with $h$ when $h_{\mathrm{c} 1}<h<h_{\mathrm{c} 2}$ (in phase II).

\subsection{Kinetic energy}

From (1) and (40) we have an exact relation between the kinetic energy of the electrons $E_{\text {kin }}$ and $D$ :

$$
E_{\text {kin }}=E-U D .
$$

Otherwise, we may calculate the kinetic energy by differentiating the total energy with respect to $t$ :

$$
E_{\mathrm{kin}}=t \frac{\partial E}{\partial t} .
$$

We calculate $E_{\text {kin }}$ in the exact theory by resolving the corresponding integral equations (see (B1)-(B5) in reference [14]—with $U$ replaced by $-|U|$ ).

In zero field $h=0$, the kinetic energy increases (algebraically) from $E_{\text {kin }}=$ $-4 t \sin (n \pi / 2) / \pi$ at $U=0$ (see section 4.1) with increasing $|U| / t$ for both signs of $U$ (figure 6) and for $n=1$ it is a symmetric function of $U / t$. The magnetic field facilitates the transition

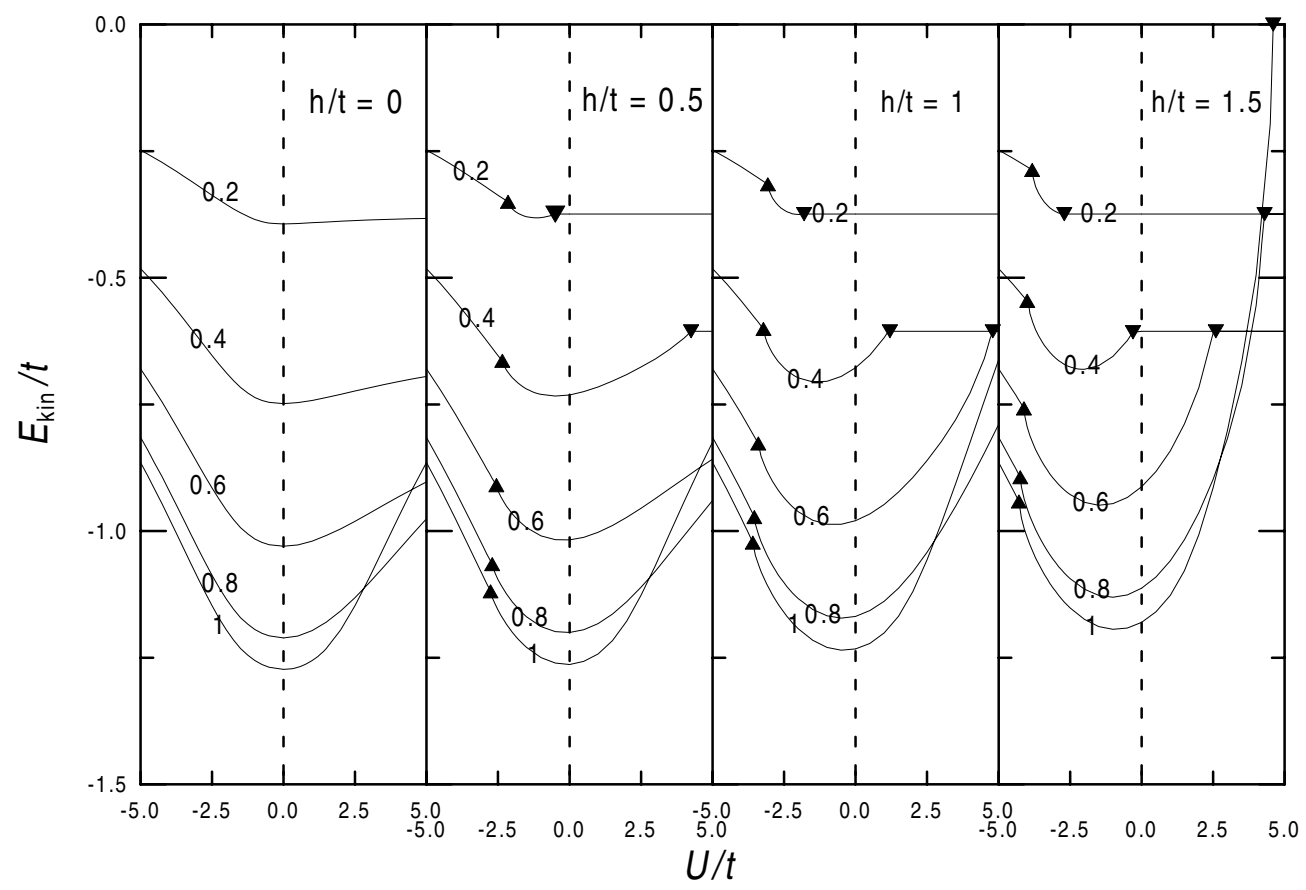

Figure 6. The ground-state kinetic energy $E_{\text {kin }}$ versus $U / t$. The notation is the same as for figure 2 . 
into a fully saturated spin state with constant $E_{\text {kin }}$ at $U>U_{\mathrm{c} 2}$. In finite magnetic field, $E_{\mathrm{kin}} / t$ decreases with $U / t$ only in phase I (zero spin), $U \leqslant U_{\mathrm{c} 1}$ (to the left of the upward-pointing solid triangles in figure 6). For strong coupling $|U| / t \gg\left|U_{\mathrm{c} 1}\right|(U<0)$, we have $s=0$ and $E_{\text {kin }} \propto-t^{2} /|U|$ (see equation (A.19) and equation (A.4)). This circumstance shows that the local electron pairs at the limit of large $-U / t$ have a bandwidth of the order of the exchange parameter of the effective pseudo-spin Hamiltonian [28] rather than a bandwidth $\sim t$ as in the weak-coupling limit $|U| / t \ll 1$.

In the intermediate phase II $\left(U_{\mathrm{c} 1}<U<U_{\mathrm{c} 2}, 0<s<n / 2\right)$, the behaviour of $E_{\mathrm{kin}}$ versus $U$ is in general non-monotonic (the region between the downward- and upward-pointing triangles in figure 6) and in this phase the kinetic energy approaches its minimum value.

Finally, in phase III, $U \geqslant U_{\mathrm{c} 2}(s=n / 2)$, the kinetic energy is constant. In this case $E_{\mathrm{kin}}=-2 t \sin (n \pi) / \pi$, so $E_{\mathrm{kin}}$ has the same value for $n$ and $1-n$. This circumstance is clearly shown for the curves for $h / t=1.5$ and $n=0.2,0.8$ or $n=0.4,0.6$ to the right of the downward-pointing solid triangles in figure 6. In particular, for $n=1$ and $s=1 / 2$ (or $n=s=0)$ we have $E_{\text {kin }}=0$.

The behaviour of $E_{\mathrm{kin}} / t$ versus $h / t$ is non-monotonic, similarly to the dependence of $E_{\mathrm{kin}} / t$ on $U / t$. In a weak magnetic field $h \leqslant h_{\mathrm{c} 1}$, the magnetization is zero (phase I) and $E_{\text {kin }}$ is constant. In a stronger magnetic field $h_{\mathrm{c} 1} \leqslant h \leqslant h_{\mathrm{c} 2}$ (phase II), the kinetic energy decreases with $h$ and passes through its minimum value. Further increase of $h$ leads to the occupation of higher energy states in the conduction band and a corresponding increase of the kinetic energy. In sufficiently strong field $h>h_{\mathrm{c} 2}$ (the spin saturation phase), $E_{\text {kin }}$ is a constant. It is symmetric relative to quarter-filling and has the same value for $n$ and $1-n$. Unpaired electrons inside the narrow band at $U<0$ are nearly localized $(D \approx 0)$ and the suppression of $\left|E_{\text {kin }}\right|$ down to zero by the applied field near half-filling resembles a trivial metal-insulator transition at $U \geqslant 0$ for vanishing carrier concentration [14].

\subsection{Chemical potential and compressibility}

The chemical potential can be derived from the system of Lieb-Wu equations, using the exact relation (see appendix D)

$$
\mu=\frac{\partial E}{\partial n}
$$

Over a broad range of $h$ and for all $n, \mu$ increases (algebraically) with increasing $U / t$ (figure 7), similarly to in the $h=0$ case [14].

At $n=1$ and $U<0$, the chemical potential is equal to $U / 2$ for arbitrary magnetic field. It is field independent also in the case of an empty band $(n=0)$. However, at $U>0, \mu$ is field dependent at relatively strong $h$. Note that $\mu / t$ versus $U / t$ shows sharp transitions near the spin onset and spin saturation (figure 7).

On the other hand, $\mu(U)$ versus $U / t$ in a weak field $h \leqslant h_{\mathrm{c} 1}$ for all $n \neq 1$ displays a smooth crossover from the BCS-like itinerant behaviour $(\mu \geqslant n U / 2-2 t)$ to the Bose condensation regime of local pairs $(\mu \leqslant n U / 2-2 t)$ when the renormalized chemical potential $\bar{\mu} \equiv \mu-n U / 2$ approaches the bottom of the conduction band [14]. The finite shift of the chemical potential $(\mu=U / 2)$ at half-filling is characteristic of the asymmetric Hubbard model in equation (1). In a slightly modified attractive Hubbard model with particle-hole symmetry [37], this shift is equal to zero at half-filling. As one can see from figure 7, the variation of $\mu$ versus $U$ at $n=1$ and $n=0$ for $U \leqslant 0$ is independent of $h$. We found in addition that the chemical potential in the presence of a magnetic field at $U \leqslant 0$ in the vicinity of $n=1$ changes smoothly as $h$ changes, in contrast to its discontinuity in the repulsive Hubbard model at half-filling [8]. Such behaviour shows the gapless character of the charge energy excitations for all $h$ and $U \leqslant 0$ 


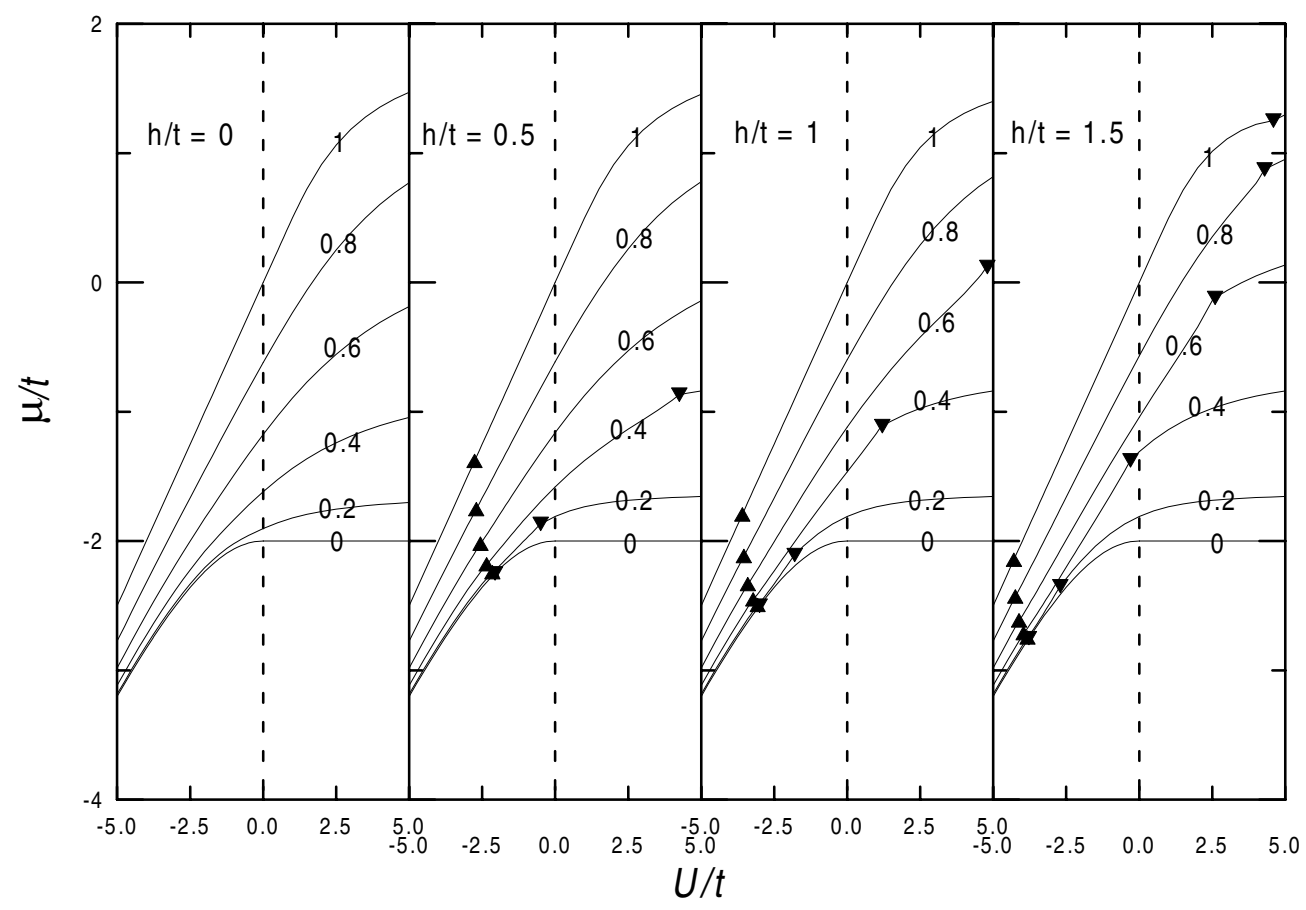

Figure 7. The ground-state chemical potential $\mu / t$ versus $U / t$. The notation is the same as for figure 2 .

at half-filling. Note that the absence of a charge gap in one dimension is in contrast with the findings from Monte Carlo calculations for an attractive two-dimensional Hubbard model at half-filling [38, 39].

The chemical potential is field independent in phases I and III. In the intermediate phase II $\left(h_{\mathrm{c} 1}<h<h_{\mathrm{c} 2}\right)$ for all $0<n<1$, the field dependence of $\mu$ is monotonic, so we have the lower and the upper bounds for $\mu$ at any fixed $U<0$, valid for arbitrary values of $h$ and $n$ :

$$
-\sqrt{U^{2} / 4+4 t^{2}} \leqslant \mu \leqslant U / 2 \text {. }
$$

Using the set of integral equations, we calculated the behaviour of the compressibility $\kappa_{\mathrm{ch}} \equiv \partial \mu / \partial n$ (see (E1)-(E5) in reference [14] with $U$ replaced by $-|U|$ ).

In the absence of a magnetic field, $\kappa_{\mathrm{ch}}$ increases with $U$ for all $n \neq 1$ (figure 8). Exactly at half-filling, $\kappa_{\mathrm{ch}}$ shows a discontinuity at $U / t=0: \kappa_{\mathrm{ch}}=t \pi$ at $U \rightarrow-0$ and $\kappa_{\mathrm{ch}}=0$ for $U>0$. In a relatively strong magnetic field, $\kappa_{\mathrm{ch}}$ may behave non-monotonically in phase II $(0<s<n / 2)$ and approaches its maximum value in the positive- $U$ region for $n>1 / 2$. At $U<0,|U| / t \gg 1$, we have $\kappa_{\mathrm{ch}} \approx t^{2} \pi^{2} /|U|$ for all $h$ (see equation (24)), consistent with the corresponding result for $\chi$ at $U>0$ (see section 6.5) [9,21]. For all $U<0$ and $h$, the compressibility $\kappa_{\mathrm{ch}}$ is maximal at half-filling. In the presence of a magnetic field, at the spin onset (upward-pointing triangles in figure 8) and near spin saturation (downward-pointing triangles in figure 8$)$, there is a cusp (sharp transition) in $\kappa_{\mathrm{ch}}$. In the fully saturated phase $\left(U \geqslant U_{\mathrm{c} 2}\right)$ we have $\kappa_{\mathrm{ch}}=2 t \pi^{2} \sin (n \pi)$, so $\kappa_{\mathrm{ch}}$ has the same value for $n$ and $1-n$ (figure 8 , to the right of the downward-pointing triangles). In this phase the compressibility is maximal at $n=1 / 2$. In particular, $\kappa_{\mathrm{ch}}=0$ at $U \geqslant U_{\mathrm{c} 2}$ in an empty or half-filled band (incompressible Fermi liquid, consisting of fully polarized electrons) and this is similar to the Mott-Hubbard localization of electrons at $n=1$ and $U>0$ in the critical field. 


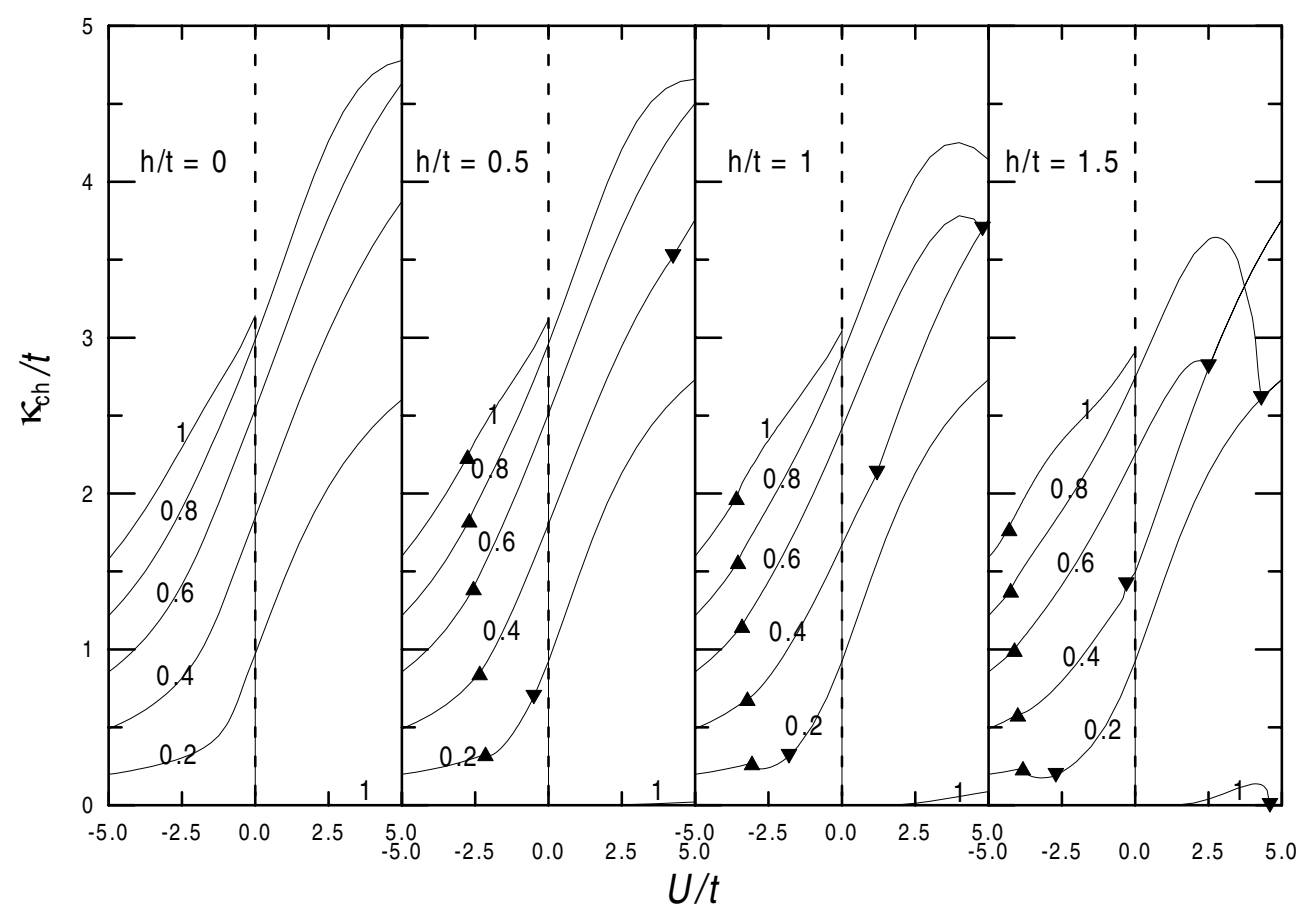

Figure 8. The ground-state compressibility $\kappa_{\mathrm{ch}}$ versus $U / t$. The notation is the same as for figure 2 .

For fixed $U$ at $h \leqslant h_{\mathrm{c} 1}$ and $h \geqslant h_{\mathrm{c} 2}$, the compressibility is independent of $h$. In the intermediate phase $h_{\mathrm{c} 1} \leqslant h \leqslant h_{\mathrm{c} 2}$, the compressibility shows slightly non-monotonic behaviour near $n=1$ as the magnetic field increases. For non-interacting electrons $(U=0)$, $\kappa_{\text {ch }}$ decreases with $h$ for all $n$ (see equation (19)).

Note that the compressibility with a single maximum as a function of $U / t$ for different $n$ qualitatively reproduces the main features of $E_{\text {kin }}$ shown in figure 6.

Using the analytical results for the ground-state energy of the half-filled band [11], we can calculate $\kappa_{\mathrm{ch}}$ near the spin onset:

$$
\left.\kappa_{\mathrm{ch}}\right|_{n \rightarrow 1}=\frac{t \pi I_{1}(-2 \pi t / U)}{I_{0}(-2 \pi t / U)}+\frac{t s \pi I_{1}(-2 \pi t / U)}{I_{0}^{2}(-2 \pi t / U)}
$$

where $I_{0}$ and $I_{1}$ are modified Bessel functions. Our numerical results coincide with this formula. For the charge susceptibility $\kappa_{\mathrm{ch}}^{-1}$, this result is consistent with the corresponding zero-field spin susceptibility $\chi$ in the repulsive Hubbard model $[9,21]$.

The problem of determining the spectrum of the charge-density-wave excitations at halffilling as a function of $s$ essentially does not differ from the analogous problem of determining the spin-density-wave (SDW) spectrum as a function of electron concentration in the repulsive Hubbard model with $h=0$ [16]. Note that the velocity of the charge excitations for the $U<0$ case, $v_{\mathrm{ch}}$, simply reproduces the charge susceptibility $v_{\mathrm{ch}}=\pi / 2 \kappa_{\mathrm{ch}}[10,21]$.

\subsection{Spin (magnetic) susceptibility}

In phases I and III the average spin $s$ does not depend on $h$, i.e., the spin susceptibility $\chi \equiv t \partial s / \partial h$ is always equal to zero. Thus the curves for $\chi^{-1}$ versus $U / t$ (figure 9) exist only in the intermediate phase $U_{\mathrm{c} 1} \leqslant U \leqslant U_{\mathrm{c} 2}$ with spin $0<s<n / 2$ (between the upward- and 


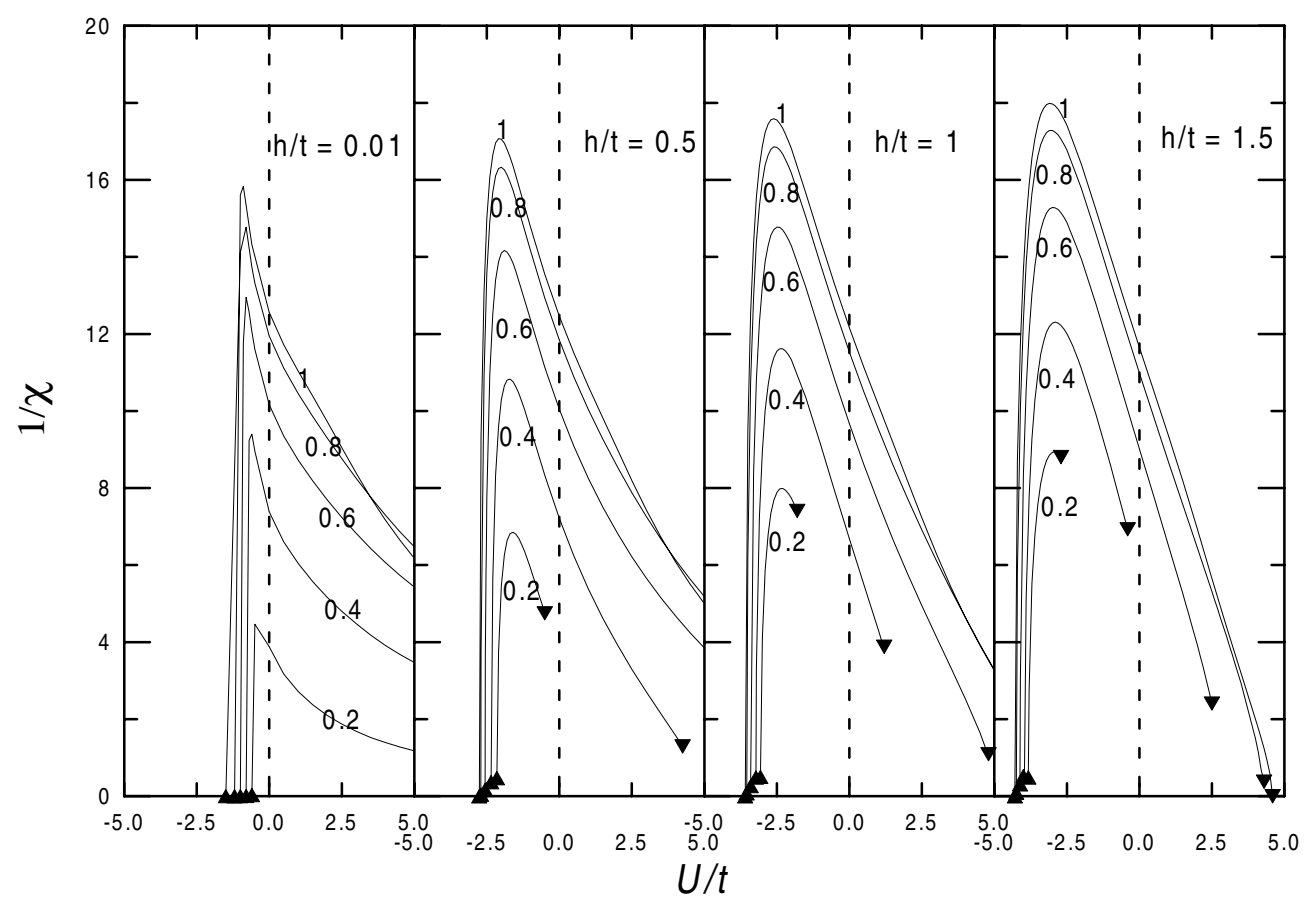

Figure 9. The ground-state inverse spin susceptibility $\chi^{-1}$ versus $U / t$. The notation is the same as for figure 2. Curves exist only between downward- and upward-pointing triangles. Beyond this region, $\chi=0$.

downward-pointing triangles). Note that the spin onset (downward triangles) in the presence of a field takes place for finite $\chi^{-1}$ for all $n \neq 1$, in full agreement with our previous results (see section 5.1). In an infinitesimal field $h \rightarrow 0, \chi^{-1}$ becomes non-analytic at $U=0$ and the curves for all $0<n \leqslant 1$ have a discontinuity at $U / t \rightarrow 0$.

The reverse spin (magnetic) susceptibility $\chi^{-1}$ shows a rapid increase in the $U<0$ region for various $n$ and $h$. This is obvious evidence for the existence of a spin energy gap and suppression of spin excitations by the increasing of a coupling constant, $|U|$. When $h \rightarrow 0$, $\chi^{-1}$ exists only for $U>0$. For $U>0$, there is no spin energy gap $\left(h_{\mathrm{c} 1}=0\right)$ for all $U$ and $\chi^{-1}$ decreases smoothly with $U / t$ and approaches the spin (magnetic) saturation at $U_{\mathrm{c} 2}$ (downward-pointing triangles). For finite $h$-values, $\chi^{-1}$ versus $U / t$ has a maximum in the negative- $U$ region (figure 9).

At $U \rightarrow U_{\mathrm{c} 2}$ and $U \rightarrow U_{\mathrm{c} 1}, \chi^{-1} \rightarrow 0$ ( $\chi$ diverges $)$ for $n=1$ and $\chi^{-1} \neq 0(\chi$ is finite $)$ for all $n \neq 1$.

Enhancement of $\kappa_{\mathrm{ch}}$ and $\chi$ has the same physical origin: the high density of states near the top of the conduction band.

For fixed $U / t$ and $n$, the curves for $\chi^{-1}$ versus $h / t$ exist only in the intermediate phase $h_{\mathrm{c} 1} \leqslant h \leqslant h_{\mathrm{c} 2}$. At $h \rightarrow h_{\mathrm{c} 1}$ and $h \rightarrow h_{\mathrm{c} 2}$, we have $\chi^{-1} \rightarrow 0$ for $n=1$ and $\chi^{-1} \neq 0$ for all $n \neq 1$.

\section{Summary}

In this paper we have calculated the exact ground-state properties in the one-dimensional Hubbard model for arbitrary sign of $U$. We have derived the analytical expressions at $U=0$ 
and the expansions in powers of $t /|U|$ and performed numerical calculations of the groundstate properties for various electron concentrations $n$ over a wide range of coupling strength $U / t$ and magnetic field $h / t$.

Several interesting features emerge from the investigation of the ground-state properties under the variation of $U / t$ for various $n$ and $h$. All of the thermodynamic quantities for various band fillings $n$ are continuous functions of $h / t$ and $U / t$, including $U=0$, although they are not analytical functions and exhibit sharp transitions (kinks) at the onset of magnetization and spin (magnetic) saturation. The calculated phase diagram in the $U-n$ plane shows two consecutive second-order phase transitions near the onset of magnetization and the saturation.

The total energy, the magnetization and the concentration of doubly occupied sites are monotonic functions of both $U / t$ and $h / t$ over the whole range, while they are in general non-monotonic functions of the parameter $n$. On the other hand, the kinetic energy $E_{\text {kin }}$, the chemical potential $\mu$, the compressibility $\kappa_{\mathrm{ch}}$ and the spin (magnetic) susceptibility $\chi$ show non-monotonic behaviour as functions of $U / t, h / t$ and $n$. We find that $\mu$ is a monotonic function of $U$ and it also decreases with $n$ at given $U$ and $h$. We find a close connection between the presence of the spin energy gap in the spectrum and enhanced spin (magnetic) susceptibility as a function of $h / t$ at $h \rightarrow h_{\mathrm{c} 1}$ for $U<0$ and the formation of a charge energy gap and enhanced compressibility at half-filling for the $U>0$ model.

For all $n=1, E_{\text {kin }}$ in the absence of a magnetic field is a symmetric function of $U / t$ and shows a smooth crossover from the weakly bound electronic states with $E_{\mathrm{kin}} \sim t$ to the strong-coupling regime with $E_{\mathrm{kin}} \sim t^{2} /|U|$, as coupling strength $|U| / t$ is increased. Note that at large $|U| / t(U<0)$ and $s=0$, the free-single-particle motion of electrons inside the band is strongly suppressed by increasing $|U|$ due to the large occupation of doubly occupied sites in the bound state.

In the absence of a magnetic field, $\chi^{-1}$ becomes non-analytic at $U=0$ and the curves for $\chi^{-1}$ versus $U$ for different $n$ show discontinuity at $U / t \rightarrow 0$. The compressibility $\kappa_{\text {ch }}$ away from half-filling $(n \neq 1)$ increases with $U$ for the entire parameter space. However, at $n=1$ it shows a discontinuity at $U=0$ from its non-interacting value to $\kappa_{\mathrm{ch}}=0$. In some range of $U>0, \kappa_{\mathrm{ch}}$ becomes identically zero independently of $h$. Note that $\kappa_{\mathrm{ch}}$ is a non-monotonic function of $n$ at $U>0$ and decreasing function of $U$ at $U<0$.

In addition, we found that the charge energy gap for all $h$ and $U \leqslant 0$ does not exist and the chemical potential $\mu$ exhibits a smooth change with $n$ in the vicinity of $n=1$. Our numerical and analytical calculations of $\mu$ for $n=1$ and $n=0$ provide the corresponding upper and lower bounds for $\mu$ for the entire $U$-space. Work currently in progress is aimed to calculate the variation of the exact ground-state charactersitics versus concentration and magnetic field for various $U>0$ and $U<0$ in the entire parameter space of $0 \leqslant n \leqslant 1$ and wide range of $h>0$ respectively.

\section{Acknowledgments}

The authors would like to acknowledge the National Science Council of the ROC for support under the grant NSC89-2112-M-032-010. ANK is greatly indebted to Professor W F Pong for the invitation to visit Tamkang University and the hospitality extended to him during his visit there. 


\section{Appendix A. Strong-coupling expansion}

We introduce the expansions up to the second order of the parameter $4 t /|U| \ll 1$ :

$$
\begin{aligned}
& \rho(k)=\frac{1}{2 \pi}-\frac{4 t}{U} \rho_{1}(k)+\left(\frac{4 t}{U}\right)^{2} \rho_{2}(k)+\cdots \\
& \sigma(\lambda)=-\frac{4 t}{U} \sigma_{1}(l)+\left(\frac{4 t}{U}\right)^{2} \sigma_{2}(l)+\cdots \\
& Q=Q_{0}+\frac{4 t}{U} Q_{1}+\left(\frac{4 t}{U}\right)^{2} Q_{2}+\cdots
\end{aligned}
$$

where $l \equiv-4 t \lambda / U$.

Substituting these series into the Lieb-Wu equations (see section 3 in [14] and section 3 in this paper) and taking the terms of the same order of magnitude, we have

$$
Q_{0}=U(1-2 s) \pi \Theta(-U)+n \pi \Theta(U) .
$$

$\Theta(x)=1$ if $x>0$ and $\Theta(x)=0$ if $x<0$, and

$$
\begin{aligned}
& \rho_{1}(k)=\frac{\cos k}{\pi} \int_{-b}^{b} \sigma_{1}(l) \tilde{f}_{1}(k, l) \mathrm{d} l \\
& \sigma_{1}(l)=\frac{1}{2 \pi^{2}} \int_{-Q}^{Q} \tilde{f}_{1}(k, l) \mathrm{d} k-\frac{2}{\pi} \int_{-b}^{b} \sigma_{1}\left(l^{\prime}\right) \tilde{f}_{2}\left(l, l^{\prime}\right) \mathrm{d} l^{\prime} \\
& \int_{-Q_{0}}^{Q_{0}} \rho_{1}(k) \mathrm{d} k=\frac{Q_{1}}{\pi} \\
& \int_{-b}^{b} \sigma_{1}(l) \mathrm{d} l=\frac{n}{2}-s .
\end{aligned}
$$

where $b \equiv 4 t B /|U|$ and

$$
\begin{aligned}
& \tilde{f}_{1}(k, l) \equiv\left(\frac{U}{4 t}\right)^{2} f_{1}(k, \lambda)=\frac{1}{1+[l-(4 t /|U|) \sin k]^{2}} \\
& \tilde{f}_{2}\left(l, l^{\prime}\right) \equiv\left(\frac{U}{4 t}\right)^{2} f_{2}\left(\lambda, \lambda^{\prime}\right)=\frac{1}{4+\left(l-l^{\prime}\right)^{2}} .
\end{aligned}
$$

We can ignore the term $(4 t /|U|) \sin k$ in the denominator of $\tilde{f}_{1}(k, l)$ in equations (A.5) and (A.6) without loss of accuracy. Thus we have

$$
\begin{aligned}
& \rho_{1}(k)=c_{1} \cos k \\
& c_{1}=\frac{1}{\pi} \int_{-b}^{b} \frac{\sigma_{1}(l)}{1+l^{2}} \mathrm{~d} l \\
& Q_{1}=2 \pi c_{1} \sin Q_{0} \\
& \sigma_{1}(l)=\frac{Q_{0}}{\pi^{2}\left(1+l^{2}\right)}-\frac{2}{\pi} \int_{-b}^{b} \frac{\sigma_{1}\left(l^{\prime}\right)}{4+\left(l-l^{\prime}\right)^{2}} \mathrm{~d} l^{\prime} .
\end{aligned}
$$

The function $\sigma_{1}(l)$ can be calculated using the integral equation (A.14) along with the normalization condition (A.8). At spin (magnetic) saturation $s=n / 2$, we have $b=0$ and $\sigma_{1}(l)=Q_{0} /\left(1+l^{2}\right) \pi^{2}$. In particular, at $U<0$ and half-filling $(n=1, s=1 / 2)$, we have $\sigma_{1}(l)=0$. 
Using the expression obtained for $\sigma_{1}(l)$, we can calculate $c_{1}$ from equation (A.12). The coefficient $c_{1}$ monotonically increases from zero at $s=n / 2$ with increasing $n$ from 0 up to 1 or by decreasing $s$ from $n / 2$ down to 0 .

Thus we have

$$
E=\left(\frac{n}{2}-s\right) \Theta(-U)+E_{\mathrm{p}}
$$

where

$$
\begin{aligned}
& E_{\mathrm{p}}=-\frac{2 t}{\pi} \sin Q_{0}-\frac{t^{2}}{|U|}\left(e_{1}+\mathcal{O}\left(\frac{t}{U}\right)\right) \\
& e_{1}=4 c_{1}\left[2 Q_{0}-\sin \left(2 Q_{0}\right)\right] \\
& D=\left(\frac{n}{2}-s\right) \Theta(-U)+\frac{t}{U} \mathcal{O}\left(\frac{t}{U}\right) \\
& E_{\mathrm{kin}}=-\frac{2 t}{\pi} \sin Q_{0}-\frac{t^{2}}{|U|}\left(2 e_{1}+\mathcal{O}\left(\frac{t}{U}\right)\right) \\
& \mu=\frac{U}{2} \Theta(-U)-2 t \cos (n \pi) \Theta(U)+\mathcal{O}\left(\frac{t}{U}\right) \\
& \kappa_{\mathrm{ch}}=2 \pi t \sin (n \pi) \Theta(U)+\mathcal{O}\left(\frac{t}{U}\right) \\
& h=-(U+4 t \cos (2 s \pi)) \Theta(-U)+\mathcal{O}\left(\frac{t}{U}\right) \\
& \chi=8 \pi t \sin (2 s \pi) \Theta(-U)+\mathcal{O}\left(\frac{t}{U}\right) .
\end{aligned}
$$

\section{Appendix B. Magnetic field}

For the magnetic field we have

$$
h=\frac{\partial E}{\partial s}=-U \Theta(-U)-2 t \int_{-Q}^{Q} \mathrm{~d} k \rho_{s}(k) \cos k-4 t Q_{s} \rho(Q) \cos Q .
$$

$\Theta(x)=1$ if $x>0$ and $\Theta(x)=0$ if $x<0 ; \rho_{s}(k) \equiv \partial \rho(k) / \partial s$ and $\sigma_{s}(\lambda) \equiv \partial \sigma(\lambda) / \partial s$ satisfy the following integral equations:

$$
\begin{gathered}
\rho_{s}(k)=\frac{|U| \cos k}{4 t \pi} \int_{-B}^{B} \mathrm{~d} \lambda \sigma_{s}(\lambda) f_{1}(k, \lambda)+\frac{|U| \cos k}{4 t \pi}\left[f_{1}(k, B)+f_{1}(k,-B)\right] \sigma(B) B_{s} \\
\sigma_{s}(\lambda)=\frac{|U|}{4 t \pi} \int_{-Q}^{Q} \mathrm{~d} k \rho_{s}(k) f_{1}(k, \lambda)-\frac{|U|}{2 t \pi} \int_{-B}^{B} \mathrm{~d} \lambda^{\prime} \sigma_{s}\left(\lambda^{\prime}\right) f_{2}\left(\lambda, \lambda^{\prime}\right) \\
\quad+\frac{|U|}{4 t \pi}\left[f_{1}(Q, \lambda)+f_{1}(-Q, \lambda)\right] \rho(Q) Q_{s} \\
\quad-\frac{|U|}{2 t \pi}\left[f_{2}(\lambda, B)+f_{2}(\lambda,-B)\right] \sigma(B) B_{s}
\end{gathered}
$$

and $Q_{s} \equiv \partial Q / \partial s, B_{s} \equiv \partial B / \partial s$ are determined from the relations

$$
\begin{aligned}
& \int_{-Q}^{Q} \rho_{s}(k) \mathrm{d} k+2 \rho(Q) Q_{s}=-2 \Theta(-U) \\
& \int_{-B}^{B} \sigma_{s}(\lambda) \mathrm{d} \lambda+2 \sigma(B) B_{s}=-1 .
\end{aligned}
$$




\section{Appendix C. Concentration of doubly occupied sites}

For the concentration of doubly occupied sites we have

$D=\frac{\partial E}{\partial U}=\left(\frac{n}{2}-s\right) \Theta(-U)-2 \int_{-Q}^{Q} \mathrm{~d} k \rho_{U}(k) \cos k-4 Q_{U} \rho(Q) \cos Q$

where

$$
\rho_{U}(k) \equiv t \partial \rho(k) / \partial U \quad \text { and } \quad \sigma_{U}(\lambda) \equiv t \partial \sigma(\lambda) / \partial U
$$

satisfy the following integral equations:

$$
\begin{aligned}
& \rho_{U}(k)=\frac{|U| \cos k}{4 t} \int_{-B}^{B} \mathrm{~d} \lambda \sigma_{U}(\lambda) f_{1}(k, \lambda) \\
&+\frac{U \cos k}{2|U| \pi} \int_{-B}^{B} \mathrm{~d} \lambda \sigma(\lambda)\left[f_{1}(k, \lambda) / 2-(U / 4 t)^{2} f_{1}(k, \lambda)^{2}\right] \\
&+\frac{|U| \cos k}{4 t \pi}\left[f_{1}(k, B)+f_{1}(k,-B)\right] \sigma(B) B_{U} \\
& \sigma_{U}(\lambda)=\frac{|U|}{4 t \pi} \int_{-Q}^{Q} \mathrm{~d} k \rho_{U}(k) f_{1}(k, \lambda)-\frac{|U|}{2 t \pi} \int_{-B}^{B} \mathrm{~d} \lambda^{\prime} \sigma_{U}\left(\lambda^{\prime}\right) f_{2}\left(\lambda, \lambda^{\prime}\right) \\
&+\frac{U}{2|U| \pi} \int_{-Q}^{Q} \mathrm{~d} k \rho(k)\left[f_{1}(k, \lambda) / 2-(U / 4 t)^{2} f_{1}(k, \lambda)^{2}\right] \\
&-\frac{U}{|U| \pi} \int_{-B}^{B} \mathrm{~d} \lambda^{\prime} \sigma\left(\lambda^{\prime}\right)\left[f_{2}\left(\lambda, \lambda^{\prime}\right) / 2-(U / 2 t)^{2} f_{2}\left(\lambda, \lambda^{\prime}\right)^{2}\right] \\
&+\frac{|U|}{4 t \pi}\left[f_{1}(Q, \lambda)+f_{1}(-Q, \lambda)\right] \rho(Q) Q_{U} \\
&-\frac{|U|}{2 t \pi}\left[f_{2}(\lambda, B)+f_{2}(\lambda,-B)\right] \sigma(B) B_{U}
\end{aligned}
$$

and $Q_{U} \equiv t \partial Q / \partial U, B_{U}=t \partial B / \partial U$ are determined from the relations (A4) and (A5) in [14].

\section{Appendix D. Chemical potential}

For the chemical potential we have

$$
\mu=\frac{\partial E}{\partial n}=\frac{U}{2} \Theta(-U)-2 t \int_{-Q}^{Q} \mathrm{~d} k \rho_{n}(k) \cos k-4 t Q_{n} \rho(Q) \cos Q
$$

where $\rho_{n}(k) \equiv \partial \rho(k) / \partial n$ and $\sigma_{n}(\lambda) \equiv \partial \sigma(\lambda) / \partial n$ satisfy the integral equations (C2) and (C3) in [14] with $U$ replaced by $-|U|, Q_{n} \equiv \partial Q / \partial n$ is determined from the relation

$$
\int_{-Q}^{Q} \rho_{n}(k) \mathrm{d} k+2 \rho(Q) Q_{n}=\Theta(U)
$$

and $B_{n} \equiv \partial B / \partial n$ is determined by (C5) in [14].

\section{Appendix E. Spin (magnetic) susceptibility}

The spin (magnetic) susceptibility is

$$
\chi \equiv \frac{t \partial s}{\partial h} \equiv \frac{t}{h_{s}}
$$


$h_{s}=-2 t \int_{-Q}^{Q} \mathrm{~d} k \rho_{s s}(k) \cos k-8 t Q_{s} \rho_{s}(Q) \cos Q$

$$
-4 t Q_{s s} \rho(Q) \cos Q+4 t Q_{s}^{2}\left[\rho(Q) \sin Q-\left.\frac{\partial \rho(k)}{\partial k}\right|_{k=Q} \cos Q\right]
$$

where $\rho_{s}(k) \equiv \partial \rho(k) / \partial s$ was determined in appendix D of reference [14]; $\rho_{s s}(k) \equiv \partial \rho_{s}(k) / \partial s$ and $\sigma_{s s}(\lambda) \equiv \partial \sigma_{s}(\lambda) / \partial s$ satisfy the following integral equations:

$$
\begin{aligned}
& \rho_{s s}(k)=\frac{|U| \cos k}{4 t \pi} \int_{-B}^{B} \mathrm{~d} \lambda \sigma_{s s}(\lambda) f_{1}(k, \lambda)+\frac{|U| \cos k}{2 t \pi}\left[f_{1}(k, B)+f_{1}(k,-B)\right] \sigma_{s}(B) B_{s} \\
& +\frac{|U| \cos k}{4 t \pi}\left[\frac{\partial f_{1}(k, B)}{\partial B}+\frac{\partial f_{1}(k,-B)}{\partial B}\right] \sigma(B) B_{s}^{2} \\
& +\frac{|U| \cos k}{4 t \pi}\left[f_{1}(k, B)+f_{1}(k,-B)\right]\left(\left.\frac{\partial \sigma(\lambda)}{\partial \lambda}\right|_{\lambda=B}\right) B_{s}^{2} \\
& +\frac{|U| \cos k}{4 t \pi}\left[f_{1}(k, B)+f_{1}(k,-B)\right] \sigma(B) B_{s s} \\
& \sigma_{s s}(\lambda)=\frac{|U|}{4 t \pi} \int_{-Q}^{Q} \mathrm{~d} k \rho_{s s}(k) f_{1}(k, \lambda)-\frac{|U|}{2 t \pi} \int_{-B}^{B} \mathrm{~d} \lambda^{\prime} \sigma_{s s}\left(\lambda^{\prime}\right) f_{2}\left(\lambda, \lambda^{\prime}\right) \\
& +\frac{|U|}{2 t \pi}\left[f_{1}(Q, \lambda)+f_{1}(-Q, \lambda)\right] \rho_{s}(Q) Q_{s} \\
& -\frac{|U|}{t \pi}\left[f_{2}(\lambda, B)+f_{2}(\lambda,-B)\right] \sigma_{s}(B) B_{s} \\
& +\frac{|U|}{4 t \pi}\left[\frac{\partial f_{1}(Q, \lambda)}{\partial Q}+\frac{\partial f_{1}(-Q, \lambda)}{\partial Q}\right] \rho(Q) Q_{s}^{2} \\
& +\frac{|U|}{4 t \pi}\left[f_{1}(Q, \lambda)+f_{1}(-Q, \lambda)\right]\left(\left.\frac{\partial \rho(k)}{\partial k}\right|_{k=Q}\right) Q_{s}^{2} \\
& +\frac{|U|}{4 t \pi}\left[f_{1}(Q, \lambda)+f_{1}(-Q, \lambda)\right] \rho(Q) Q_{s s} \\
& -\frac{|U|}{2 t \pi}\left[\frac{\partial f_{2}(\lambda, B)}{\partial B}+\frac{\partial f_{2}(\lambda,-B)}{\partial B}\right] \sigma(B) B_{s}^{2} \\
& -\frac{|U|}{2 t \pi}\left[f_{2}(\lambda, B)+f_{2}(\lambda,-B)\right]\left(\left.\frac{\partial \sigma(\lambda)}{\partial \lambda}\right|_{\lambda=B}\right) B_{s}^{2} \\
& -\frac{|U|}{2 t \pi}\left[f_{2}(\lambda, B)+f_{2}(\lambda,-B)\right] \sigma(B) B_{s s}
\end{aligned}
$$

and $Q_{s} \equiv \partial Q / \partial s, B_{s} \equiv \partial B / \partial s$ were given in appendix D of reference [14]; $Q_{s s} \equiv \partial Q_{s} / \partial s$ and $B_{s s} \equiv \partial B_{s} / \partial s$ are determined from the relations

$$
\begin{aligned}
& \int_{-Q}^{Q} \rho_{s s}(k) \mathrm{d} k+4 \rho_{s}(Q) Q_{s}+2\left(\left.\frac{\partial \rho(k)}{\partial k}\right|_{k=Q}\right) Q_{s}^{2}+2 \rho(Q) Q_{s s}=0 \\
& \int_{-B}^{B} \sigma_{s s}(\lambda) \mathrm{d} \lambda+4 \sigma_{s}(B) B_{s}+2\left(\left.\frac{\partial \sigma(\lambda)}{\partial \lambda}\right|_{\lambda=B}\right) B_{s}^{2}+2 \sigma(B) B_{s s}=0 .
\end{aligned}
$$

The Lieb-Wu equations (see section 3 in [14] and section 3 in this paper) and (E.1)-(E.6) determine $\chi$ as a function of $-U / t, n$ and $s$. 


\section{References}

[1] Dagotto E 1994 Rev. Mod. Phys. 66763

[2] Anderson P W 1987 Science 2351196

[3] Emery V J and Kivelson S A 1993 Physica C 209597

Emery V J and Kivelson S A 1995 Nature 374434

[4] Emery V J and Kivelson S A 1998 J. Phys. Chem. Solids 591705

[5] Emery V J, Kivelson S A and Tranquada J M 2000 Proc. Natl Acad. Sci. USA at press

[6] Tranquada J M 1997 Physica B 241745

[7] Nakano T, Oda M, Manabe C, Momono N, Miura Y and Ido M 1994 Phys. Rev. B 4916000

[8] Lieb E H and Wu F Y 1968 Phys. Rev. Lett. 201445

[9] Takahashi M 1969 Prog. Theor. Phys. 421098

Takahashi M 1970 Prog. Theor. Phys. 431619

Takahashi M 1970 Prog. Theor. Phys. 44348

[10] Krivnov V Ya and Ovchinnikov A A 1975 Sov. Phys.-JETP 40781

[11] Bahder T B and Woynarovich F 1986 Phys. Rev. B 332114

[12] Lee K-J-B and Schlottmann P 1988 Phys. Rev. B 3811566 Lee K-J-B and Schlottmann P 1989 Phys. Rev. B 409104

[13] Marsiglio F 1997 Phys. Rev. B 55575

[14] Kocharian A N, Yang C and Chiang Y L 1999 Phys. Rev. B 597458

[15] Shiba H 1972 Phys. Rev. B 6930

[16] Coll C F III 1974 Phys. Rev. B 92150

[17] Frahm H and Korepin V E 1990 Phys. Rev. B 4210553

[18] Kawakami N and Okiji A 1990 Prog. Theor. Phys. Suppl. 101429

[19] Usuki T, Kawakami N and Okiji A 1989 Phys. Lett. A 135476

[20] Ovchinnikov A A 1970 Sov. Phys.-JETP 301160

[21] Takahashi M 1974 Prog. Theor. Phys. 52103

[22] Woynarovich F 1983 J. Phys. C: Solid State Phys. 166593

[23] Schlottmann P 1997 Int. J. Mod. Phys. B 11355

[24] Ha Z N C 1996 Quantum Many-Body Systems in One Dimension vol 12 (Singapore: World Scientific)

[25] Sutherland B 1985 Springer Lecture Notes in Physics vol 242 (Berlin: Springer) p 1

[26] Kocharian A N, Yang C, Chiang Y L and Kioussis N 1999 Physica B 259-261 739

[27] Shiba H 1972 Prog. Theor. Phys. B 482171

[28] Emery V J 1976 Phys. Rev. B 142989

[29] Beni G, Holstein T and Pincus P 1973 Phys. Rev. B 8312

[30] Carmelo J and Baeriswyl D 1988 Phys. Rev. B 377541

[31] Kocharian A N, Jermakian A K and Yang C 1997 Physica B 230-232 918

[32] Hirsch J E 1991 Physica C 179317

[33] Griffiths R B 1964 Phys. Rev. 133 A768

[34] Yang C N and Yang C P 1966 Phys. Rev. 150327 Yang C N and Yang C P 1966 Phys. Rev. 151258

[35] Kocharian A N and Sebold J 1996 Phys. Rev. B 5312804

[36] Press W H, Teukolsky S A, Vetterling W T and Flannery B P 1992 Numerical Recipes in Fortran 2nd edn (Cambridge: Cambridge University Press)

[37] dos Santos R R 1994 Phys. Rev. B 50635

[38] Singer J M, Pedersen M H, Schneider T, Beck H and Matuttis H-G 1996 Phys. Rev. B 541286

[39] Singer J M, Schneider T and Pedersen M H 1998 Eur. Phys. J. B 217 